\title{
Enhanced MALDI-TOF MS Analysis of Phosphopeptides Using an Optimized DHAP/DAHC Matrix
}

\author{
Junjie Hou, ${ }^{1,2}$ Zhensheng Xie, ${ }^{1}$ Peng Xue, ${ }^{1}$ Ziyou Cui, ${ }^{1,2}$ Xiulan Chen,, ${ }^{1,}$ Jing Li, ${ }^{1,2}$ \\ Tanxi Cai, ${ }^{1}$ Peng Wu, ${ }^{1}$ and Fuquan Yang ${ }^{1}$ \\ ${ }^{1}$ Proteomic Platform and National Key Laboratory of Biomacromolecules, Institute of Biophysics, Chinese Academy of Sciences, \\ Beijing 100101, China \\ ${ }^{2}$ Department of Biology, Graduate University of the Chinese Academy of Sciences, Beijing 100049, China
}

Correspondence should be addressed to Fuquan Yang, fqyang@sun5.ibp.ac.cn

Received 30 June 2009; Revised 11 September 2009; Accepted 31 December 2009

Academic Editor: Kai Tang

Copyright (C) 2010 Junjie Hou et al. This is an open access article distributed under the Creative Commons Attribution License, which permits unrestricted use, distribution, and reproduction in any medium, provided the original work is properly cited.

\begin{abstract}
Selecting an appropriate matrix solution is one of the most effective means of increasing the ionization efficiency of phosphopeptides in matrix-assisted laser-desorption/ionization time-of-flight mass spectrometry (MALDI-TOF-MS). In this study, we systematically assessed matrix combinations of 2, 6-dihydroxyacetophenone (DHAP) and diammonium hydrogen citrate (DAHC), and demonstrated that the low ratio DHAP/DAHC matrix was more effective in enhancing the ionization of phosphopeptides. Low femtomole level of phosphopeptides from the tryptic digests of $\alpha$-casein and $\beta$-casein was readily detected by MALDI-TOF-MS in both positive and negative ion mode without desalination or phosphopeptide enrichment. Compared with the DHB/PA matrix, the optimized DHAP/DAHC matrix yielded superior sample homogeneity and higher phosphopeptide measurement sensitivity, particularly when multiple phosphorylated peptides were assessed. Finally, the DHAP/DAHC matrix was applied to identify phosphorylation sites from $\alpha$-casein and $\beta$-casein and to characterize two phosphorylation sites from the human histone $\mathrm{H} 1$ treated with Cyclin-Dependent Kinase-1 (CDK1) by MALDI-TOF/TOF MS.
\end{abstract}

\section{Introduction}

Reversible phosphorylation of serine, threonine, and tyrosine residues is one of the most common and important regulatory posttranslational modifications of proteins and plays a crucial role in many biological processes, including cellular signal transduction, regulation of enzyme activities, metabolic regulation, molecular recognition and biomolecular interaction, protein localization, cell differentiation and proliferation [1-5]. Therefore, identifying phosphorylated proteins is an important step to elucidate the structural and functional role of the protein's phosphorylation regulation.

Up to now, mass spectrometry, including matrix-assisted laser desorption/ionization time-of-flight mass spectrometry (MALDI-TOF-MS) and electrospray ionization mass spectrometry (ESI-MS) have been key technologies in characterizing protein phosphorylation and phosphoproteome, and each approach presented strengths and limitations [6-15]. However, because of the low and dynamic stoichiometry of phosphorylation on proteins $[16,17]$ and the low ionization efficiency of phosphopeptides, it remains challenging to thoroughly characterize phosphorylation sites using mass spectrometry [18]. Considerable effort has been made recently to improve the ionization efficiency of phosphopeptides by selecting and optimizing appropriate matrices with MALDI-TOF-MS. Several materials have been employed in phosphopeptide analysis, including 2, 5-dihydroxybenzoic acid (DHB) [19-21], 2, 4, 6-trihydroxyacetophenone (THAP) [22], and ionic liquid matrices [20]. In addition, ammonium salts, including diammonium hydrogen citrate (DAHC) [22-24], ammonium acetate [23] and monoammonium phosphate [25], and phosphoric acid [19-21] have been used as matrix additives and have shown some ability to enhance phosphopeptide ionization during MALDI-TOF-MS.

2, 6-Dihydroxyacetophenone (DHAP) was first used by Gorman et al. as a matrix for MALDI analysis of fragile peptides, disulfide bonding, and small proteins, including 
phosphopeptides [24]. In recent years, Xu et al. reported that the DHAP/DAHC matrix combination was used as an effective MALDI matrix in detecting methyl esterified phosphopeptides by MALDI-TOF-MS [26, 27]. After systematic optimization in this study, we found that contrary to previous reports, the matrix forms at a lower ratio of DHAP/DAHC and that this ratio shows greater efficiency in improving ionization ability of phosphopeptides. We investigated the characteristics of an optimal DHAP/DAHC matrix, and analyzed morphology and detection sensitivity of phosphopeptides. Using tryptic digests of $\alpha$ - and $\beta$-casein, we demonstrated a CID MS/MS analysis of phosphopeptides by MALDI-TOF/TOF-MS with an optimized DHAP/DAHC matrix. Finally, we used this approach to analyze phosphorylation sites on human histone $\mathrm{H} 1$ treated with CyclinDependent Kinase-1 (CDK1).

\section{Experimental}

2.1. Materials. The chemicals 2, 6-dihydroxyacetophenone (DHAP), 2, 5-dihydroxy-benzoic acid (DHB), diammonium hydrogen citrate (DAHC), ammonium bicarbonate, trifluoroacetic acid (TFA), alkaline phosphatases, $\alpha$-casein and $\beta$ casein (both from bovine milk, purity by electrophoresis) were all purchased from Sigma Aldrich (St. Louis, MO, USA). Cyclin-Dependent Kinase-1(CDK1) and human histone H1 were purchased from New England BioLabs, Inc. The standard peptides VNQIGpTLSESIK (pT, purity > 95\%) and SGSLHRIpYTHQS (pY, purity > 85\%) were synthesized in Beijing Scilight Biotechnology Ltd. Co. (Beijing, China). Sequencing grade modified trypsin (porcine) was purchased from Promega Co. (Madison, WI, USA). HPLC-grade acetonitrile and acetic acid were purchased from Mallinckrodt Baker, Inc, (Phillipsburg, NJ, USA). Ethanol and ammonium hydroxide solutions $\left(\mathrm{NH}_{3} \cdot \mathrm{H}_{2} \mathrm{O}\right)$ were purchased from Beijing Chemical Company (Beijing China); ultrapure water was obtained from a Milli-Q system (Millipore, Bedford, MA, USA). All other chemicals used were of ACS or HPLC grade.

2.2. Matrix Preparation. A $20-\mathrm{mg} / \mathrm{mL}$ dilution of DAHC stock solution was prepared with ultrapure water. The DHAP matrix stock solution was prepared by dissolving $10 \mathrm{mg}$ of DHAP in $1 \mathrm{~mL}$ of anhydrous ethanol. A series of modified DHAP/DAHC matrix solutions were prepared by mixing DHAP stock solution with DAHC stock solution at different ratios from $10: 1$ to $1: 80(\mathrm{v} / \mathrm{v})$. The $\mathrm{DHB} /$ phosphoric acid (PA) matrix was prepared as previously reported [19]; we modified this previous protocol by dissolving $20 \mathrm{mg}$ of DHB in $1 \mathrm{~mL}$ of $50 \%$ aqueous acetonitrile containing $1 \%$ phosphoric acid.

2.3. Sample Preparation. $\alpha$-Casein and $\beta$-casein $(100 \mathrm{nM}$ in $50 \mathrm{mM}$ ammonium bicarbonate buffer at $\mathrm{pH} 8.3$ ) were digested overnight at $37^{\circ} \mathrm{C}$ with trypsin at an enzyme/substrate ratio of $1: 25(\mathrm{w} / \mathrm{w})$. After digestion, TFA was added into the sample solution at $1.0 \%$ to quench the reaction. The digest was diluted with $60 \%$ acetonitrile containing $0.1 \%$ TFA to produce a series of sample solutions at concentrations of $1 \mathrm{pmol} / \mu \mathrm{L}, 200 \mathrm{fmol} / \mu \mathrm{L}, 50 \mathrm{fmol} / \mu \mathrm{L}$, $20 \mathrm{fmol} / \mu \mathrm{L}$, and $10 \mathrm{fmol} / \mu \mathrm{L}$. A stock solution containing standard peptides $\mathbf{p} \mathrm{T}(1 \mathrm{mM})$ and $\mathbf{p Y}(1 \mathrm{mM})$ was prepared using ultra pure water. The solution was diluted with $60 \%$ acetonitrile containing $0.1 \%$ TFA to $1 \mathrm{pmol} / \mu \mathrm{L}, 200 \mathrm{fmol} / \mu \mathrm{L}$, $50 \mathrm{fmol} / \mu \mathrm{L}, 20 \mathrm{fmol} / \mu \mathrm{L}$, and $10 \mathrm{fmol} / \mu \mathrm{L}$. All of the peptide solutions prepared for $\mathrm{DHB} / \mathrm{PA}$ measurements were diluted with $1 \%$ phosphoric acid.

2.4. Human Histone H1 Phosphorylation and Digestion. The phosphorylation reaction between histone $\mathrm{H} 1$ and CDK1 was carried out in vitro. Briefly, the reaction solution was prepared with $3 \mu \mathrm{L} 10 \times \mathrm{CDK} 1$ reaction buffer, $0.15 \mu \mathrm{L}$ CDK1 (150 U), $4 \mu \mathrm{L}$ histone H1 $(1 \mu \mathrm{g} / \mu \mathrm{L}), 100 \mu \mathrm{M}$ ATP, and $13 \mu \mathrm{L}$ water, then incubated at $30^{\circ} \mathrm{C}$ for 45 minutes. The phosphorylated histone $\mathrm{H} 1$ was then separated using SDS-PAGE (12\%) and stained with Coomassie Brilliant Blue using microwave-assisted methods [28]. The in-gel digestion of phosphorylated histone $\mathrm{H} 1$ was performed as follows: the band of histone $\mathrm{H} 1$ was excised from the polyacrylamide gel, washed twice with water, and destained with $40 \%$ acetonitrile/50 $\mathrm{mM} \mathrm{NH}_{4} \mathrm{HCO}_{3}$. The gel pieces were dehydrated with $100 \%$ acetonitrile and dried for 5 min using a speedvac. Disulfide bonds were reduced with DTT $\left(10 \mathrm{mM}, 56^{\circ} \mathrm{C}, 45\right.$ minutes), and the free sulfhydryl groups were alkylated with iodoacetamide $\left(55 \mathrm{mM}, 25^{\circ} \mathrm{C}\right.$, 60 minutes in the dark). Gel pieces were washed with $50 \mathrm{mM} \mathrm{NH}_{4} \mathrm{HCO}_{3}, 50 \%$ acetonitrile $/ 50 \mathrm{mM} \mathrm{NH}_{4} \mathrm{HCO}_{3}$, and dehydrated with $100 \%$ acetonitrile. After drying with a speedvac, the gel was rehydrated using $100 \mathrm{ng} / \mu \mathrm{L}$ trypsin $\left(50 \mathrm{mM} \mathrm{NH}_{4} \mathrm{HCO}_{3}, \mathrm{pH} 8.3\right.$ ) on ice for 30 minutes, and the digestion was carried out at $37^{\circ} \mathrm{C}$ for 60 minutes and then quenched with $1.0 \%$ TFA. The tryptic peptides were extracted twice with $60 \%$ acetonitrile containing $0.1 \%$ TFA, and then the combined digest solution was concentrated to $10 \mu \mathrm{L}$ under vacuum.

2.5. Sample Analysis by MALDI-TOF-MS and MALDI-TOF2MS. MALDI-TOF-MS analysis of peptides was performed using an AXIMA-CFR plus MALDI-TOF mass spectrometer (Shimadzu/Kratos, Manchester, UK) equipped with a pulsed nitrogen laser operated at $337 \mathrm{~nm}$. Positive/negative ion MALDI mass spectra were acquired in the reflectron mode under the following parameters: ion source, $20 /-20 \mathrm{kv}$, lens, $6.3 /-6.3 \mathrm{kv}$, pulsed extraction, $-2.5 / 2.5 \mathrm{kv}$, reflection, 25/-25 kV. High-energy CID MS/MS was performed with the AXIMA-TOF ${ }^{2}$ MALDI mass spectrometer (Shimadzu/Kratos, Manchester, UK), equipped with the same pulsed 337-nm nitrogen laser. Operation parameters were: ion source, $20.0 \mathrm{kV}$; lens, $6.5 \mathrm{kV}$; pulsed extraction, $-2.5 \mathrm{kV}$; and reflectron, $24.4 \mathrm{kV}$.

To prepare for MALDI-TOF-MS analysis, $1 \mu \mathrm{L}$ of peptide solution was mixed with $3 \mu \mathrm{L}$ of matrix solution in an Eppendorf tube, and $1 \mu \mathrm{L}$ of the peptide/matrix solution was spotted onto the MALDI sample plate and then crystallized either under vacuum (when DHAP/DAHC was used as matrix) or in the air (when DHB/PA was used as matrix). 


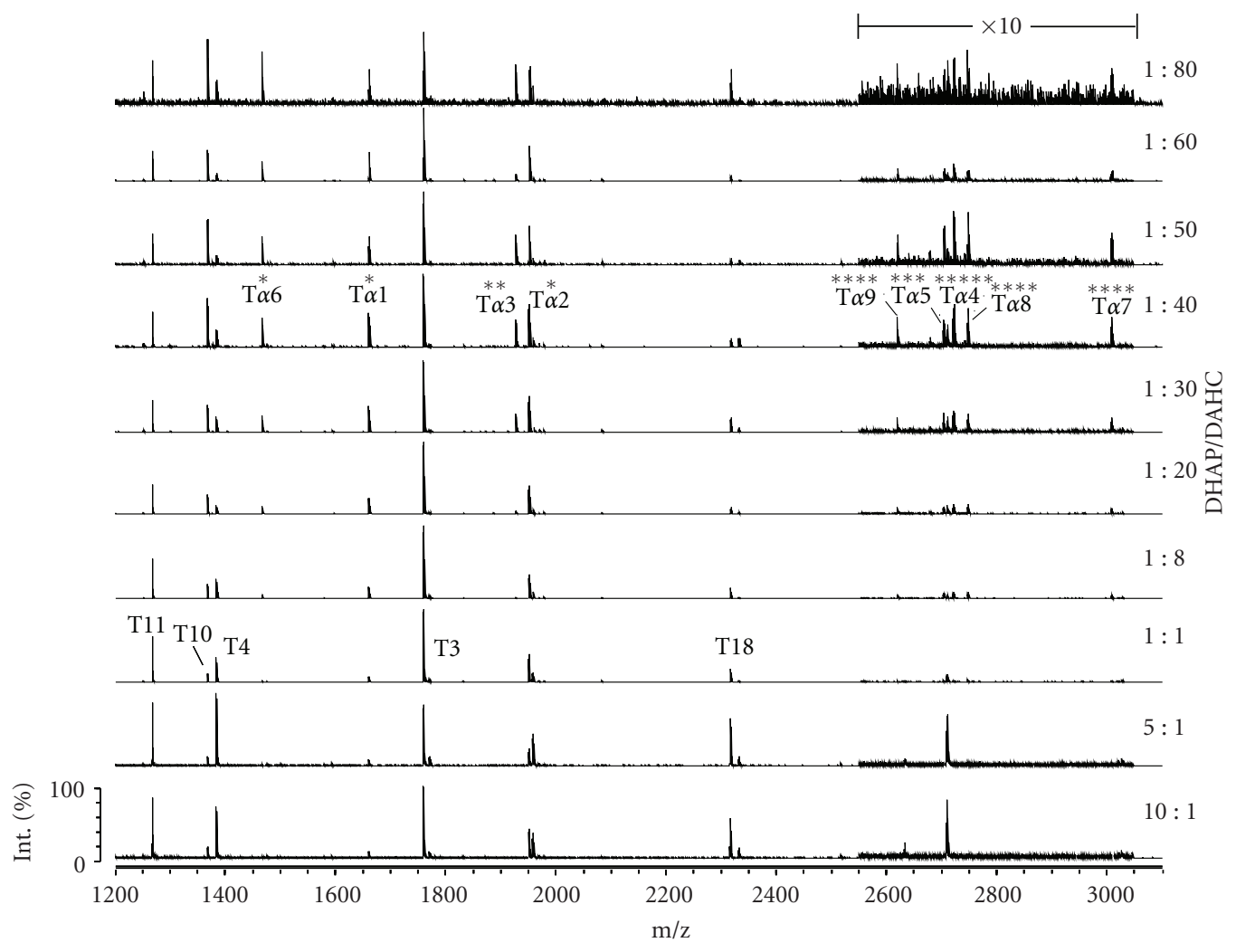

FIGURE 1: The MALDI mass spectra of the tryptic digest of $\alpha$-casein (250 fmol on target) measured in positive ion mode with DHAP/DAHC matrix at different ratios $(10: 1$ to $1: 80)$. The signal intensity is magnified 10 -fold in the $\mathrm{m} / \mathrm{z}$ range from 2575 to 3025 . The number of sites on each phosphopeptide is equal to the number of asterisks $\left(^{*}\right)$ shown.

Measurements using DHAP/DAHC were performed by detecting 15-20 different positions on the broad outer zone (see discussion below) for each sample to accumulate a total of 200 profiles ( 5 shots per profile). For measurements with $\mathrm{DHB} / \mathrm{PA}$, a total of 200 profiles (5 shots per profile) were accumulated by searching for 10-15 hot spots for each sample. All experiments were repeated three times.

The standard peptide pT (250 fmol on target) was used to determine sample homogeneity of the matrix. On each sample spot, $196(14 \times 14)$ positions were selected manually to ensure a full overview over the sample preparation. For each position, 50 profiles (5 shots per profile) were accumulated in positive ion mode. The signal intensities of $\mathrm{pT}$ in each position were recorded for further analysis.

Mass spectrometry data were processed with Launchpad 2.7.1 software (Shimadzu/Kratos, Manchester, UK). The data of the CID MS/MS spectra of phosphopeptides from histone $\mathrm{H} 1$ were compared to the SwissProt 56.6 protein sequence database (Homo sapiens) using the on-line MASCOT database search engine (Matrix Science, London, UK) with the following parameters: trypsin with three missed cleavages; average mass values; peptide mass tolerance: \pm 0.8 $\mathrm{Da}$; fragment mass tolerance: $\pm 1.0 \mathrm{Da}$; fixed modifications: Carbamidomethyl (C), variable modifications: Oxidation
(M), phosphor (ST). Only the peptides with ions score above 38 were accepted as significant matches.

\section{Results and Discussion}

3.1. Optimization of DHAP/DAHC Matrix Combination. The solution composition of the matrix is a factor that significantly influences the ionization efficiency and the quality of MALDI mass spectra [29]. Therefore, we prepared a series of different ratios of DHAP $(10 \mathrm{mg} / \mathrm{mL})$ and DAHC $(20 \mathrm{mg} / \mathrm{mL})$ matrix solutions $(10: 1,5: 1,1: 1,1: 4,1: 8$, $1: 16,1: 20,1: 30,1: 40,1: 50,1: 60$, and $1: 80 \mathrm{v} / \mathrm{v})$ and investigated these matrix combinations using the tryptic digest of $\alpha$-casein, which contains nine phosphopeptides (T $\alpha 1-\mathrm{T} \alpha 9)$ and some nonphosphopeptides (such as T4, T10, T11, T18) (see Table 1). Figure 1 shows a comparison of the MALDI-TOF-MS analysis of the tryptic digest of $\alpha$-casein (250 fmol on target) using matrix DHAP/DAHC at different ratios in positive ion mode. The signal intensity of phosphopeptides (especially for multiple phosphorylated peptides) changed dramatically with the ratio of DHAP/DAHC. It was clear that all phosphopeptides could be easily detected with a low DHAP/DAHC matrix ratio in the range of $1: 30$ to $1: 60$. In an optimal matrixsolution, the concentration 


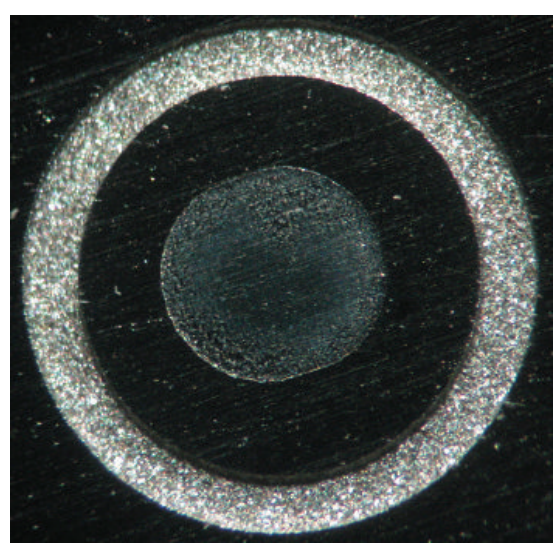

(a)

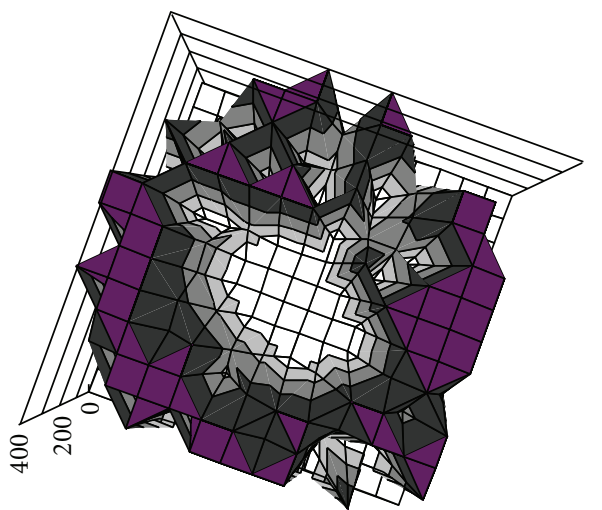

300-400

$\square$ 200-300 $\square$ 100-200

$\square 0-100$

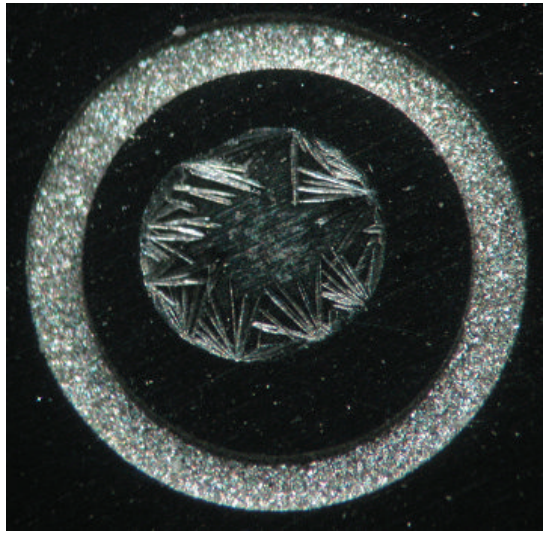

(b)

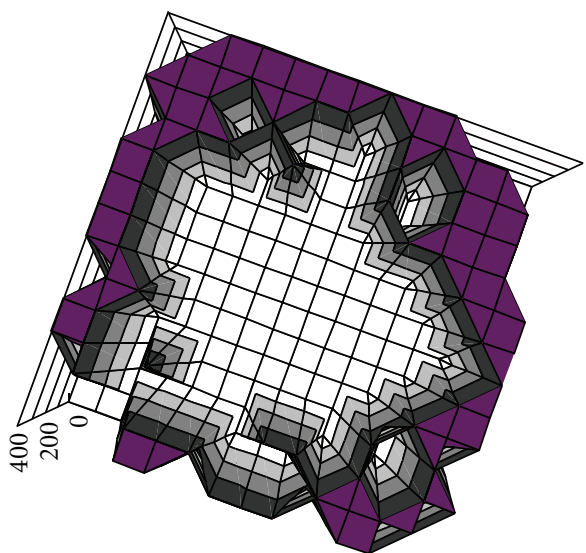

- 300-400

$\square$ 100-200

$\square 0-100$

(c)

(d)

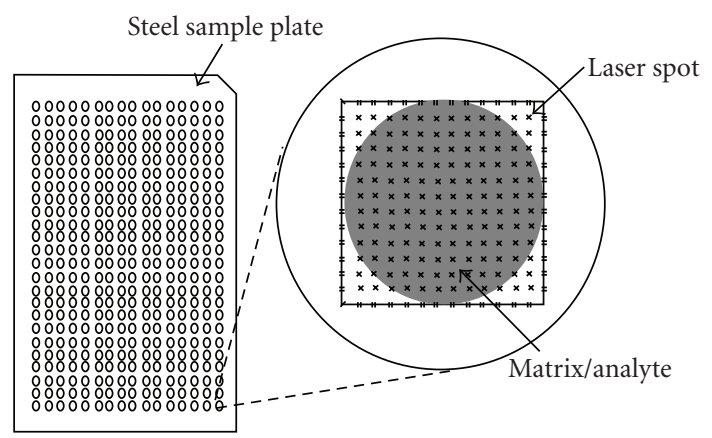

(e)

Figure 2: Top two views show the MALDI samples containing the phosphopeptide pT using (a) DHAP/DAHC matrix and (b) DHB/PA matrix. The two center pictures show the signal intensities of the phosphopeptides pT (250 fmol on target) measured with (c) DHAP/DAHC matrix and (d) DHB/PA matrix. The images only show a cut at signal intensity of 400 mv for clarity. The positions with signal intensities above $400 \mathrm{mv}$ are indicated in purple. The bottom row (e) shows a schematic of the data acquisition method for investigating sample homogeneity of the matrix. In total, 196 positions were selected as a $14 \times 14$ spots array to cover the crystalline matrix/analyte layer, and 50 profiles ( 5 shots per profiles) were accumulated at each position.

of DHAP was $1.6-3.3 \mathrm{mg} / \mathrm{mL}$, which is lower than the concentration of $10-15 \mathrm{mg} / \mathrm{mL}$ used by other groups [24, $26,27]$. Once the DHAP/DAHC ratio was down to $1: 80$, most multiple phosphorylated peptides could no longer be detected, likely because there were not sufficient numbers of
DHAP molecules to transfer the laser energy to the peptides to be protonated.

Another interesting phenomenon apparent in Figure 1: the signal intensity of nonphosphopeptides was stable (T3), enhanced (T10) or weakened (T11, T4, T18) with the 


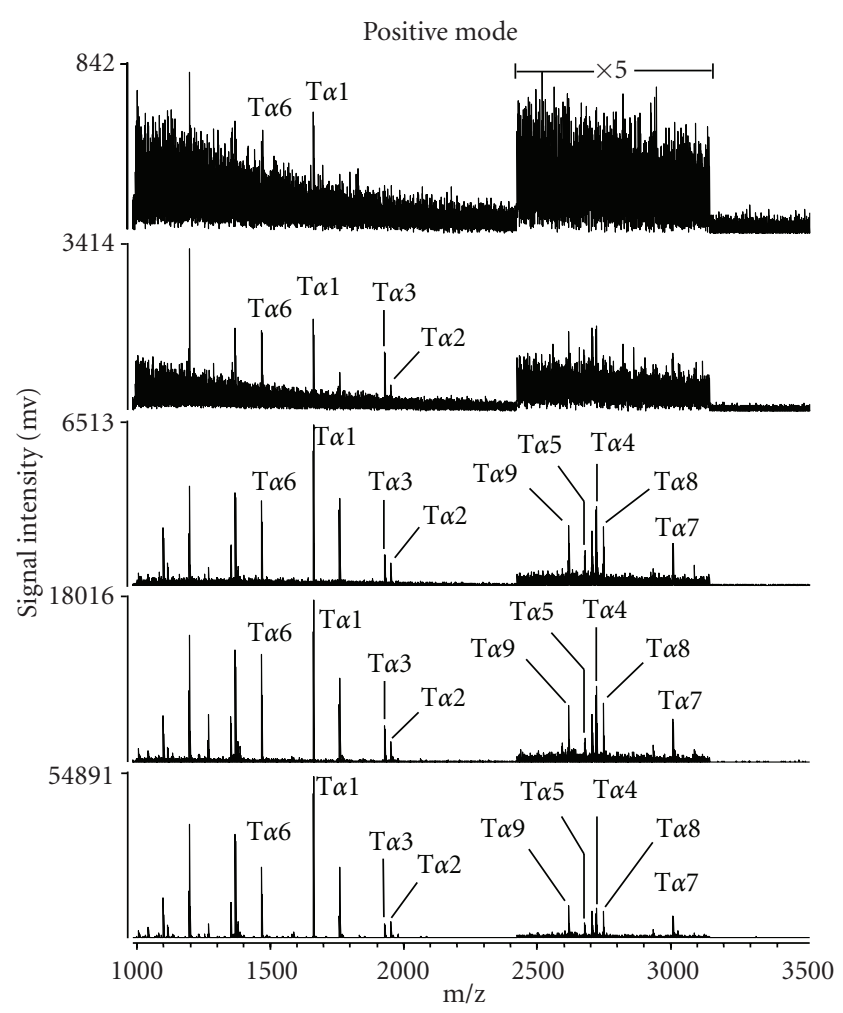

(a)

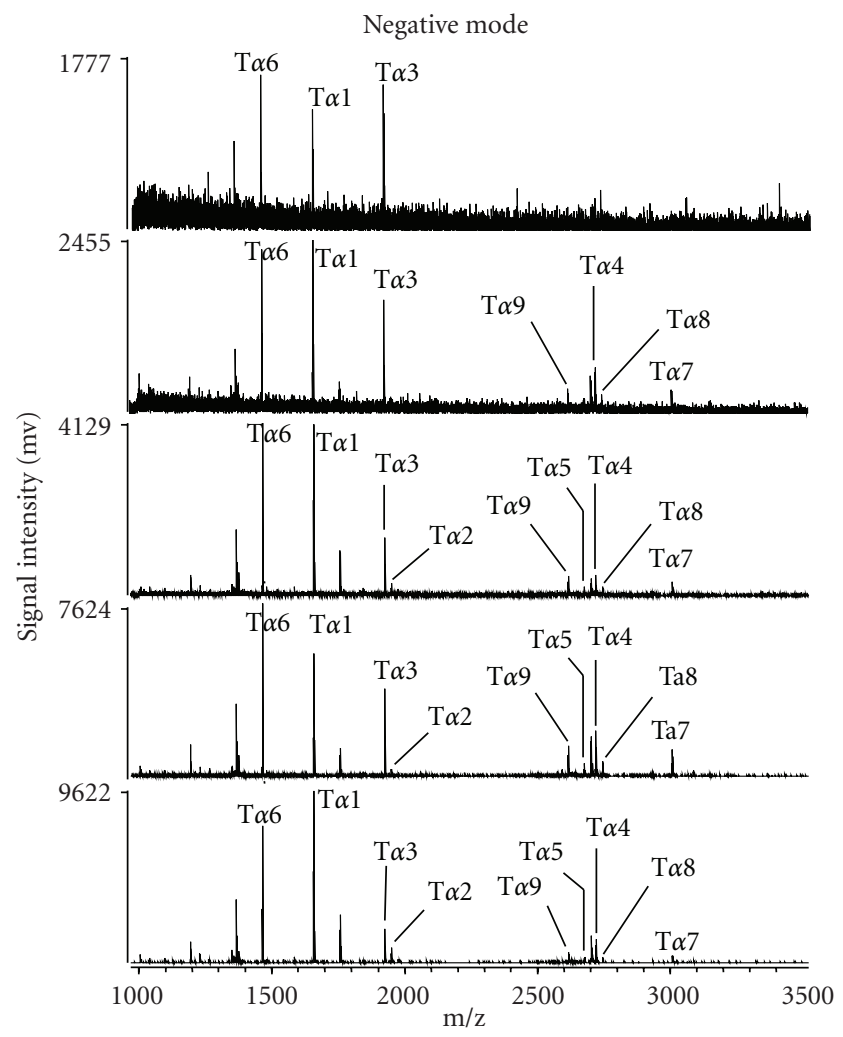

(c)

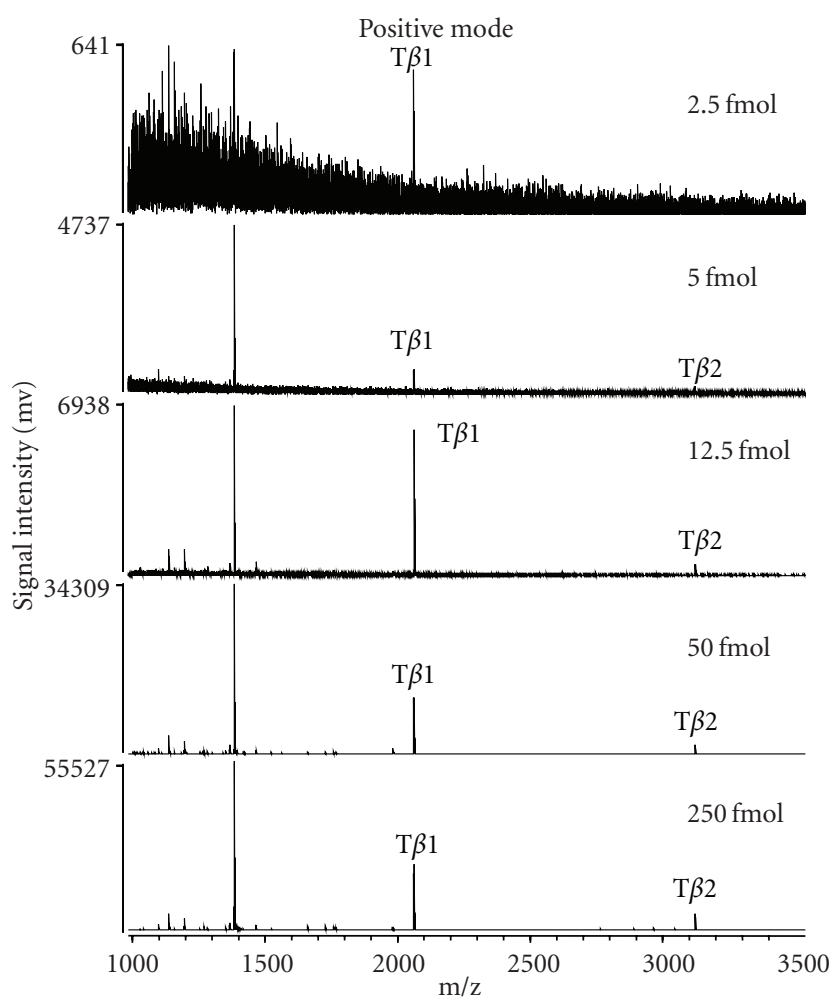

(b)

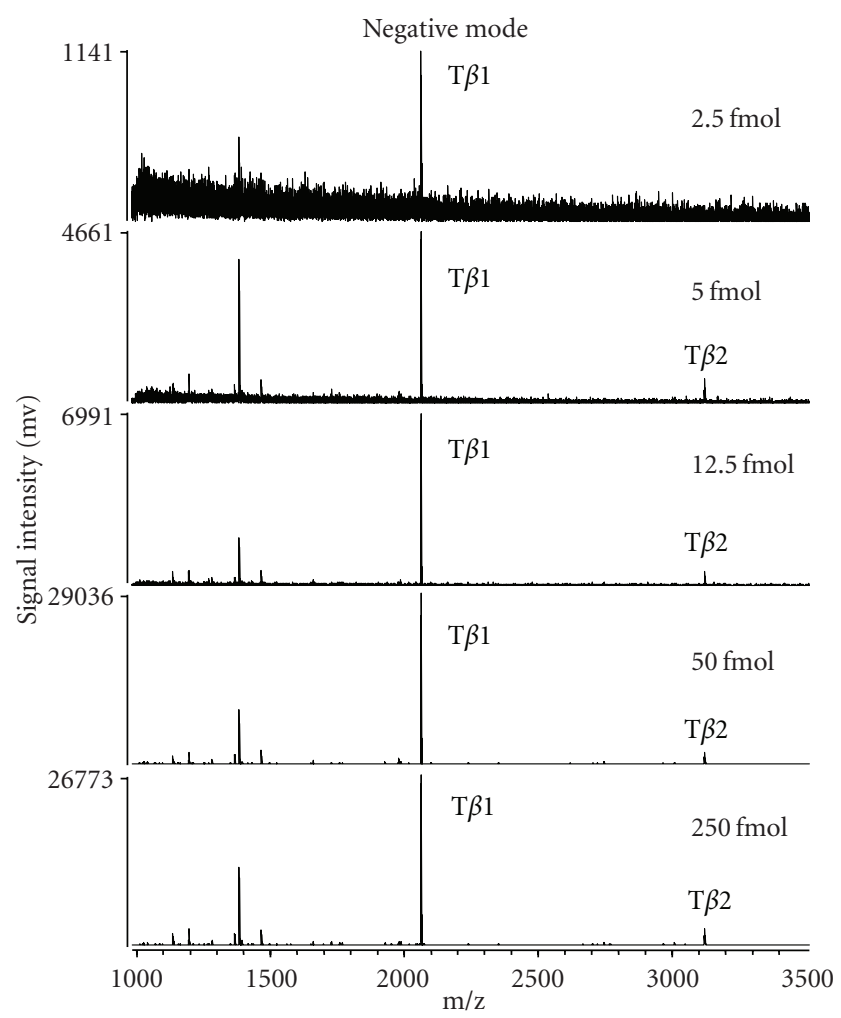

(d)

FIgure 3: The MALDI mass spectra of the tryptic digest of (a) and (c) $\alpha$-casein and (b) and (d) $\beta$-casein measured in positive (upper) and negative (lower) ion modes with the DHAP/DAHC matrix. The amount of the tryptic peptides mixture ranged from $5 \mathrm{fmol}$ to $250 \mathrm{fmol}$ on target. The signal intensity is magnified 5-fold in the $\mathrm{m} / \mathrm{z}$ range from 2400 to 3150 in section a. 


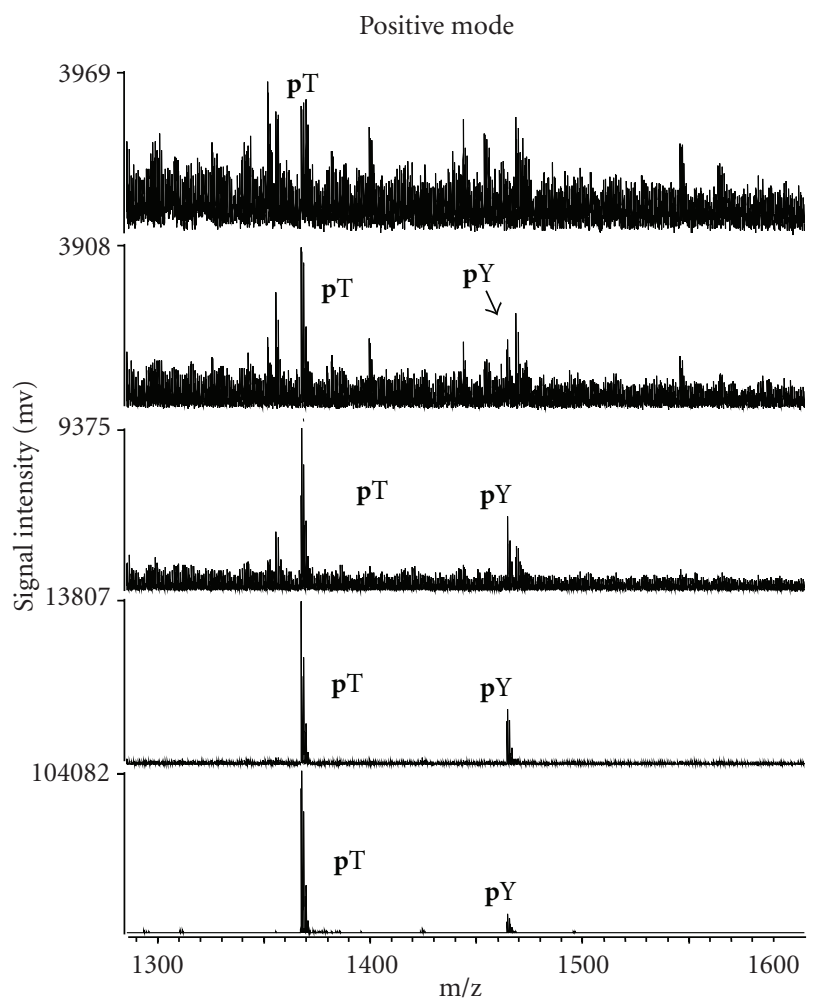

(a)

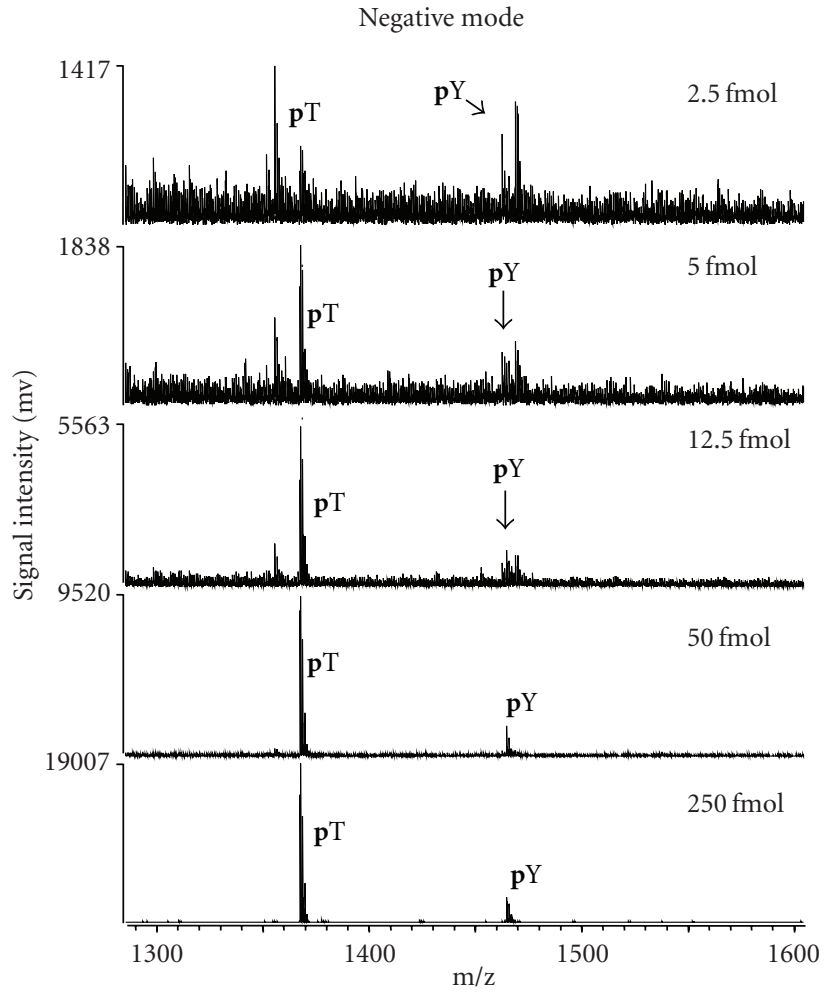

(b)

Figure 4: The MALDI mass spectra of two synthesized phosphopeptides pT and pY mixed at equimolarity from 5 to $250 \mathrm{fmol}$ on target, which were measured in positive (a) and negative (b) ion mode with DHAP/DAHC, respectively.

TABLE 1: The peptides investigated in this study*.

\begin{tabular}{|c|c|c|c|c|}
\hline Peptide ID & Sequence No. & Sequence & {$[\mathrm{M}+\mathrm{H}]_{\text {Theo }}^{+}$} & $\mathrm{B}+\mathrm{B}$ \\
\hline $\mathrm{T} \alpha 1$ & aa $106-119$ & VPQLEIVPNpSAEER & 1660.79 & 260 \\
\hline $\mathrm{T} \alpha 2$ & aa $104-119$ & KYKVPQLEIVPNpSAEER & 1951.95 & -710 \\
\hline $\mathrm{T} \alpha 3$ & aa $43-58$ & DIGpSEpSTEDQAMEDIK & 1927.68 & 3960 \\
\hline $\mathrm{T} \alpha 4$ & aa $59-79$ & QMEAEpSIpSpSpSEEIVPNpSVEQK & 2720.90 & 3680 \\
\hline $\mathrm{T} \alpha 5$ & aa $37-58$ & VNELpSKDIGpSEpSTEDQAMEDIK & 2678.02 & 3840 \\
\hline $\mathrm{T} \alpha 6$ & aa $138-149$ & TVDMEpSTEVFTK & 1466.60 & -210 \\
\hline $\mathrm{T} \alpha 7$ & aa $46-70$ & NANEEEYSIGpSpSpSEEpSAEVATEEVK & 3008.02 & 7100 \\
\hline $\mathrm{T} \alpha 8$ & aa $1-21$ & KNTMEHVpSpSpSEESIIpSQETYK & 2747.00 & 2810 \\
\hline $\mathrm{T} \alpha 9$ & aa $2-21$ & NTMEHVpSpSpSEESIIpSQETYK & 2618.91 & 2350 \\
\hline $\mathrm{T} \beta 1$ & aa $33-48$ & FQpSEEQQQTEDELQDK & 2061.82 & 6200 \\
\hline $\mathrm{T} \beta 2$ & aa $1-25$ & RELEELNVPGEIVEpSLpSpSpSEESITR & 3122.26 & -220 \\
\hline T10 & aa $81-91$ & ALNEINQFYQK & 1367.70 & -750 \\
\hline $\mathrm{T} 4$ & aa $23-34$ & FFVAPFPEVFGK & 1384.73 & -5530 \\
\hline $\mathrm{T} 3$ & aa $8-22$ & HQGLPQEVLNENLLR & 1759.59 & -590 \\
\hline T11 & aa $91-100$ & YLGYLEQLLR & 1267.71 & -6480 \\
\hline T18 & aa $132-150$ & EPMIGVNQELAYFYPELFR & 2316.14 & -6900 \\
\hline pT & - & VNQIGpTLSESIK & 1368.44 & -310 \\
\hline pY & - & SGSLHRIpYTHQS & 1465.48 & 2300 \\
\hline
\end{tabular}

* Phosphopeptides in tryptic digest of $\beta$ casein and $\alpha$ casein are denoted as $\mathrm{T} \beta 1 \sim 2$ and $\mathrm{T} \alpha 1 \sim 9$, respectively. The sites of phosphorylation are indicated with the letter " $\mathrm{p}$ " in the listed peptide sequences. T3, T4, T11, and T18 are nonphosphorylated peptide from $\alpha$ casein S1. T10 is a nonphosphorylated peptide from $\alpha$ casein S2. pT and pY are the synthesized standard phosphopeptides. $[\mathrm{M}+\mathrm{H}]^{+}$denotes the theoretical monoisotopic $m / z$ mass. "B+B" means Bull and Breese Index for peptides calculated by the Masslynx software (Waters/Micromass). 


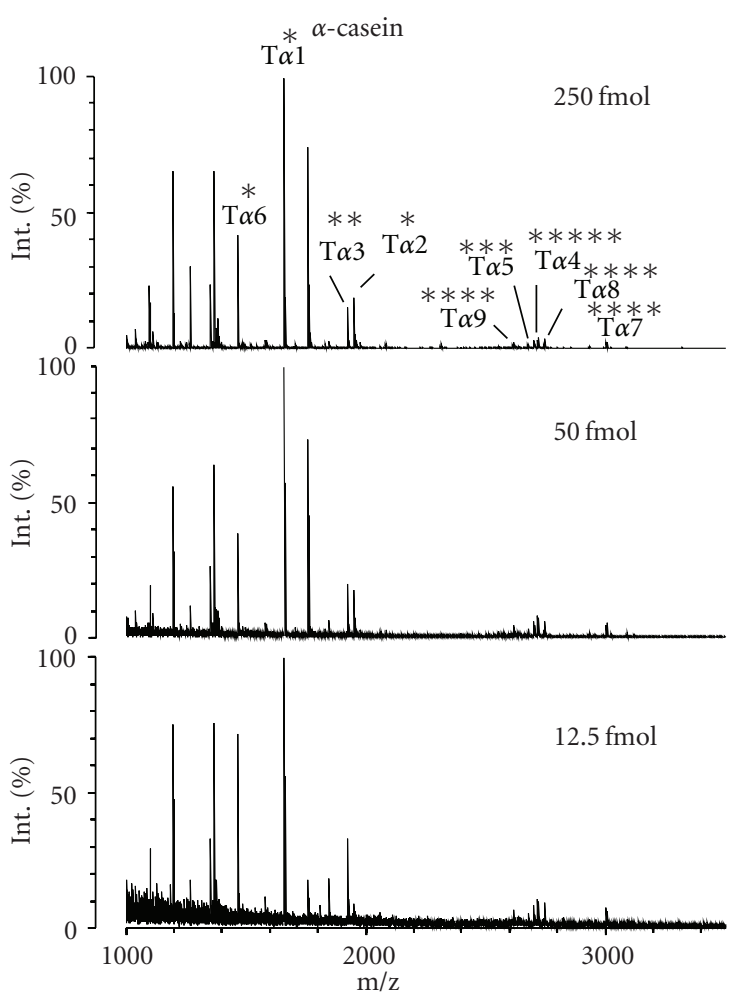

(a)

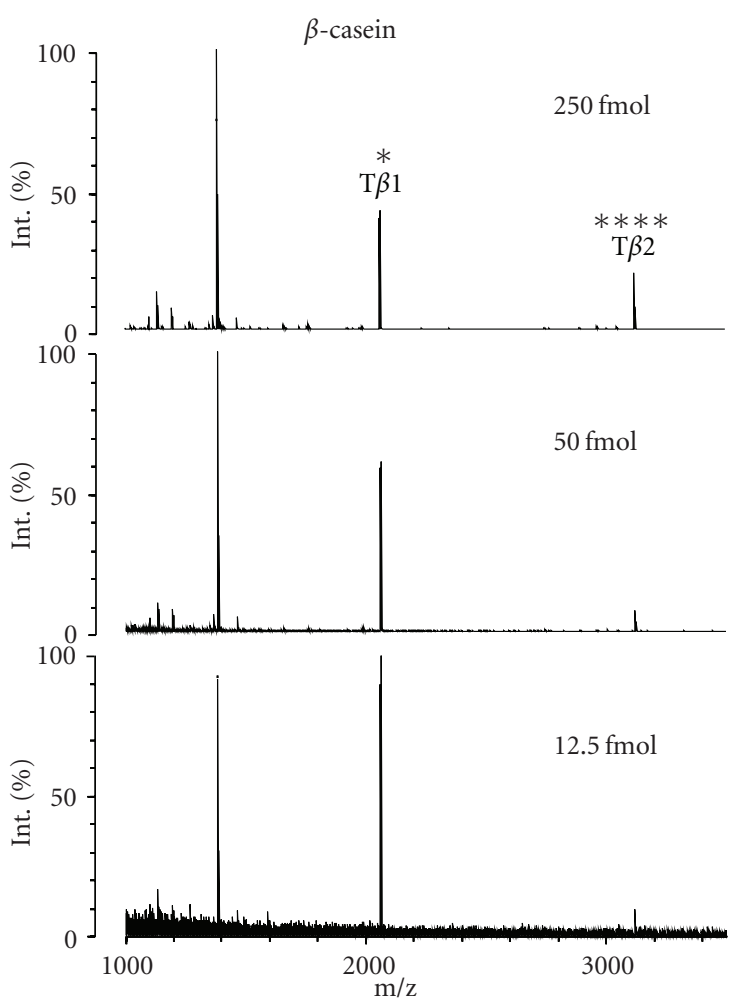

(c)

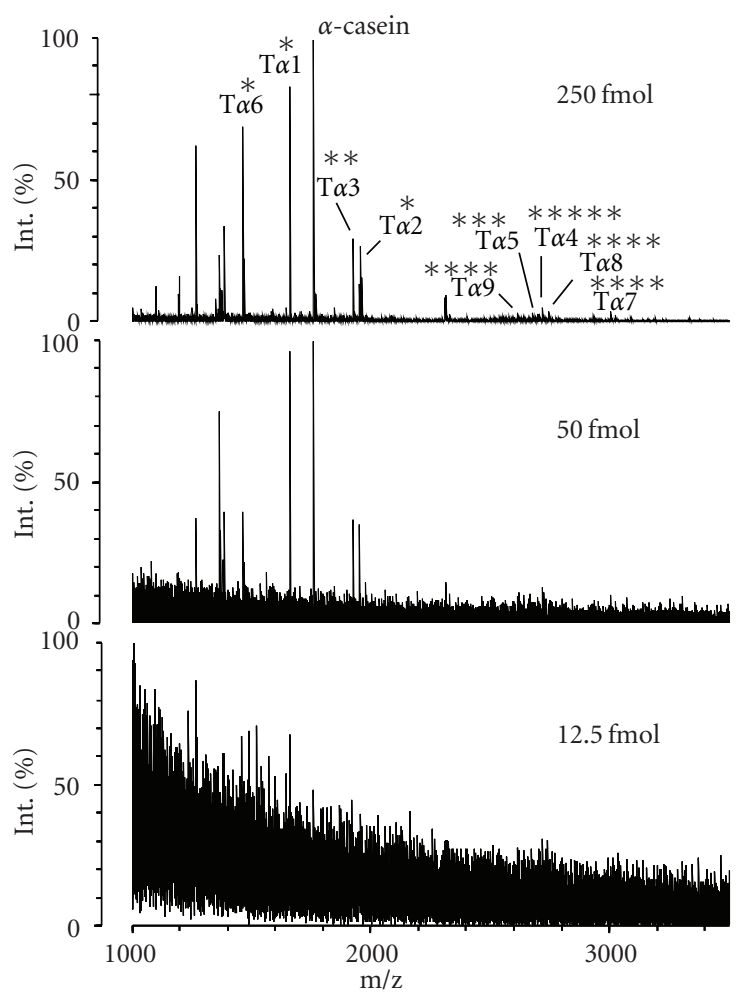

(b)

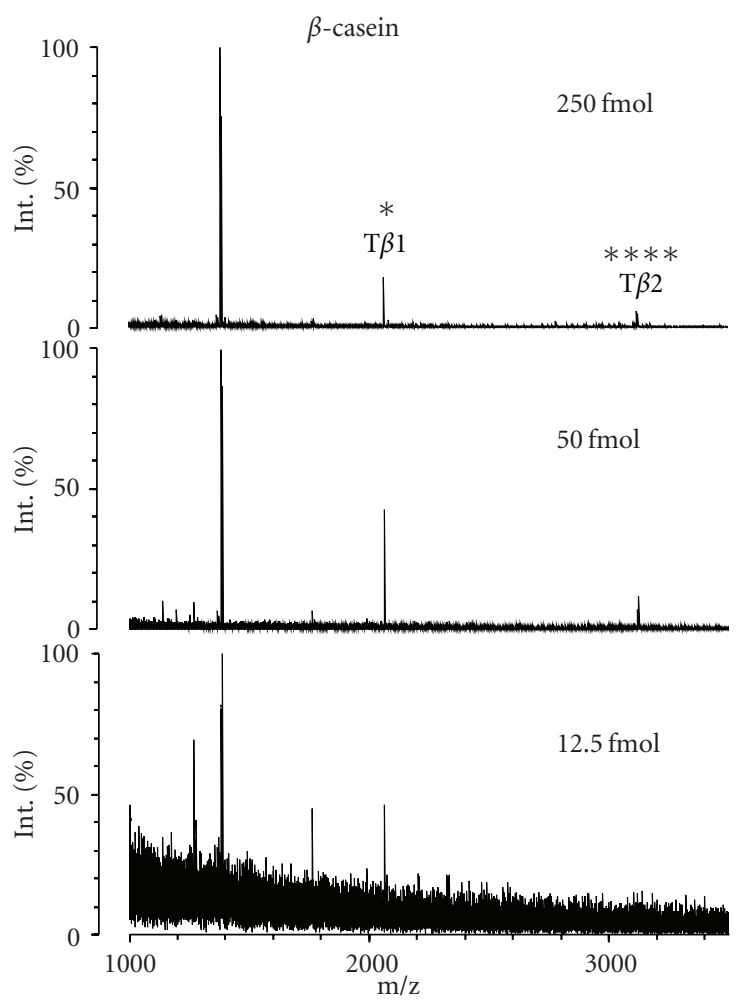

(d)

Figure 5: Tryptic digests of $\alpha$ - (upper) and $\beta$-casein (lower) (12.5-250 fmol on target) were detected with DHAP/DAHC (a) and (c) and DHB/PA (b) and (d) by MALDI-TOF MS in positive mode. DHAP/DAHC positions were selected randomly in the outer ring of the sample; for DHB/PA, only signals in hot spots were collected. In total, 200 profiles were accumulated for each sample. The number of sites on each phosphopeptide was equal to the number of asterisks $(*)$. 


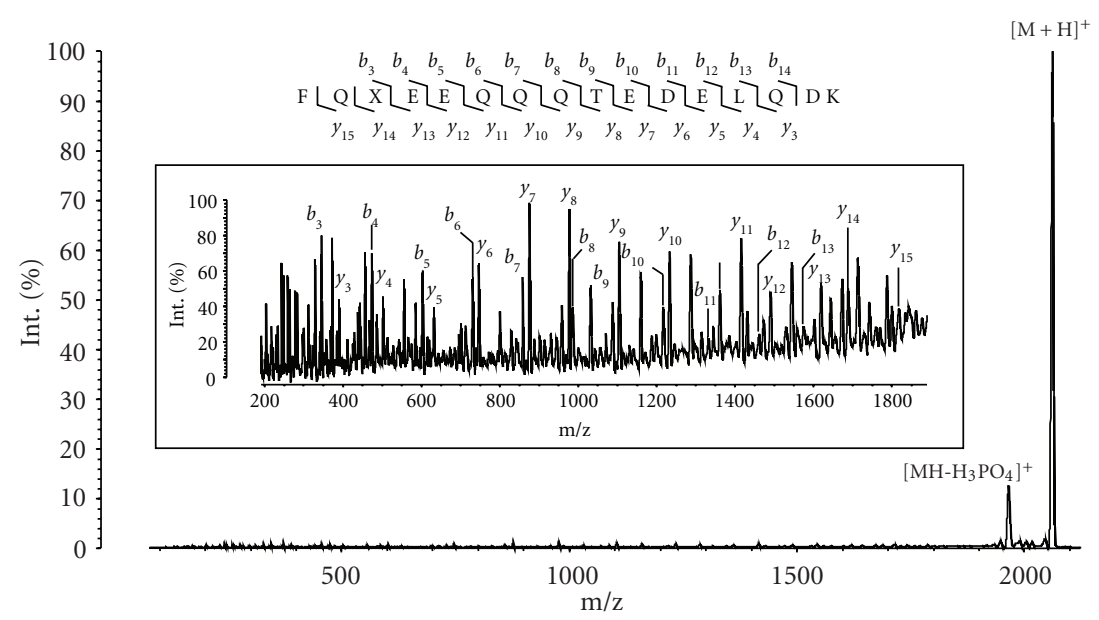

(a)

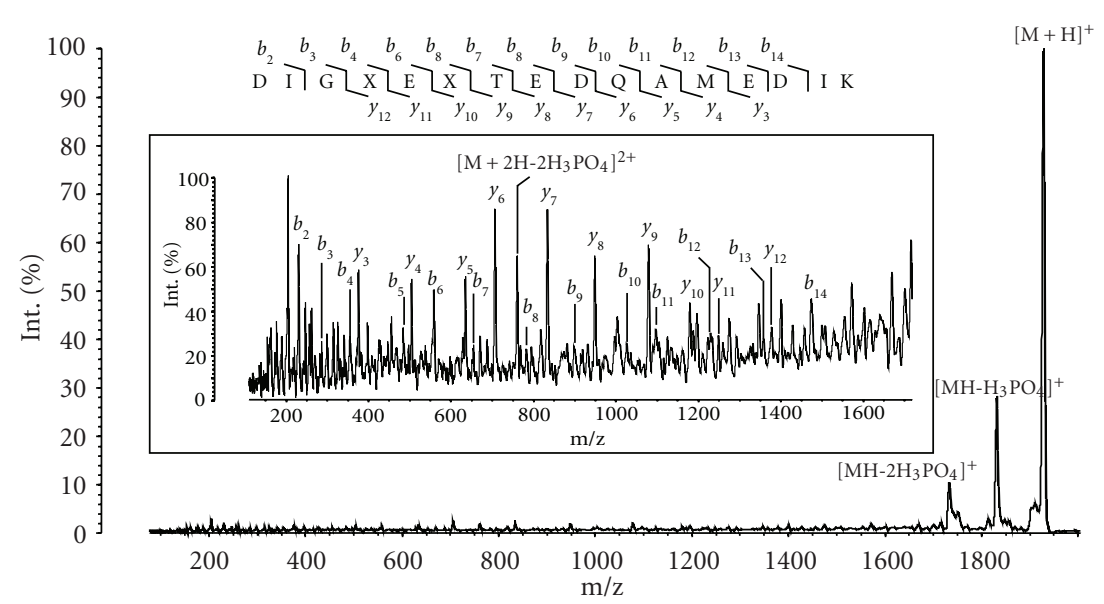

(b)

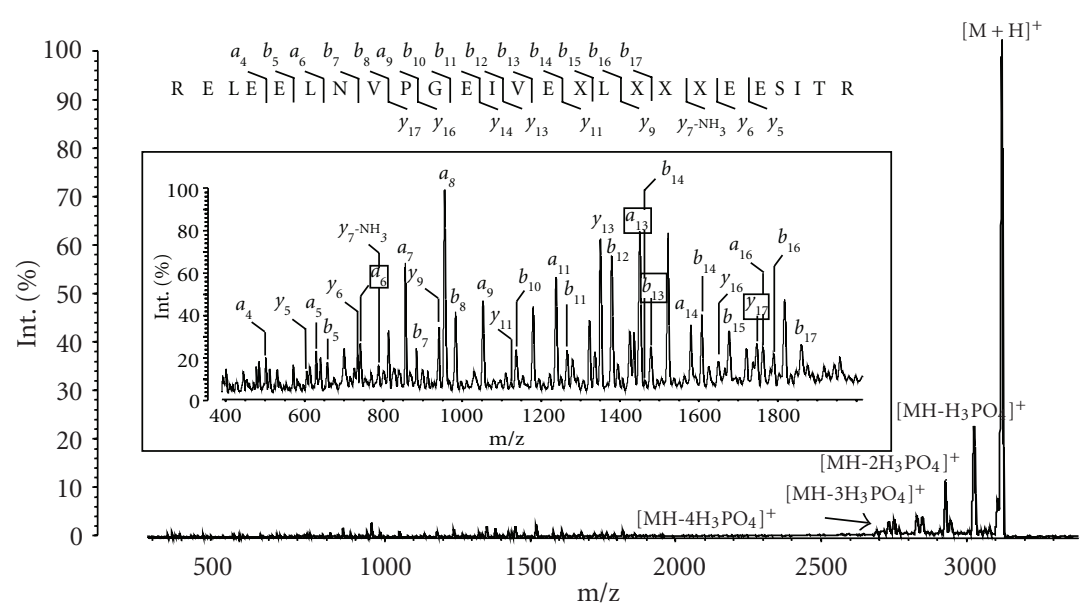

(c)

Figure 6: The MALDI MS/MS spectra of phosphopeptides (a) T $\beta 1$, (b) T $\alpha 3$, and (c) T $\beta 2$ (250 fmol for each on target) from $\alpha$-casein and $\beta$-casein were obtained by MALDI-TOF/TOF MS. The sequential losses of $\mathrm{H}_{3} \mathrm{PO}_{4}$ from the parent ions are noted. The fragment patterns of the peptides are indicated in the magnified MS/MS spectra. " $X$ " in the amino sequence shows a dehydroalanine residue converted from a phosphoserine residue by beta-elimination of $\mathrm{H}_{3} \mathrm{PO}_{4}$. 


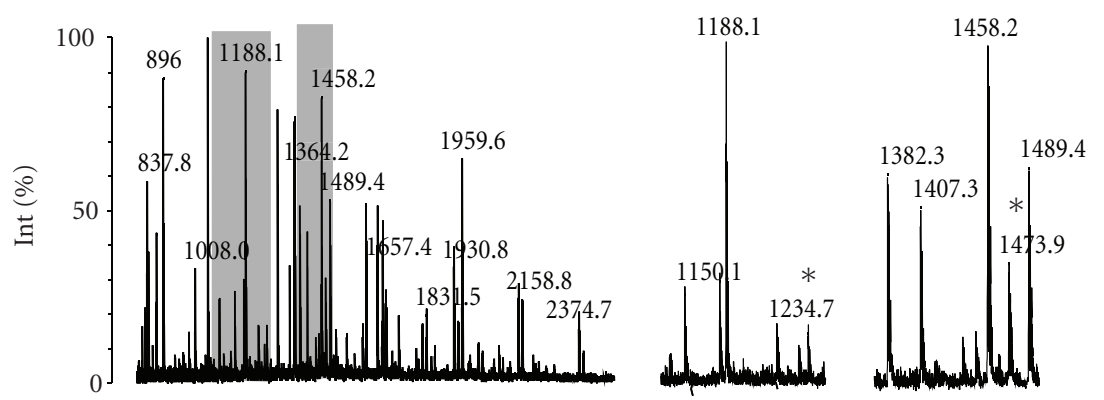

(a)
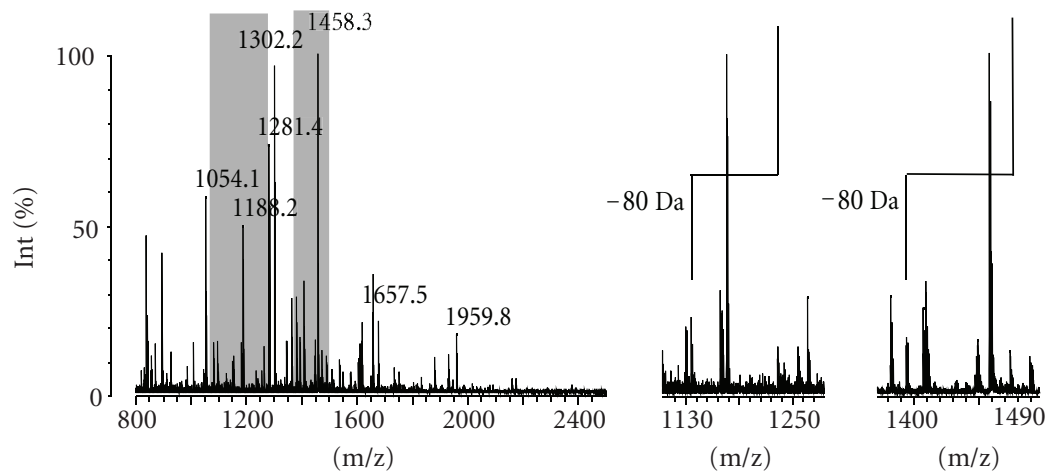

(b)

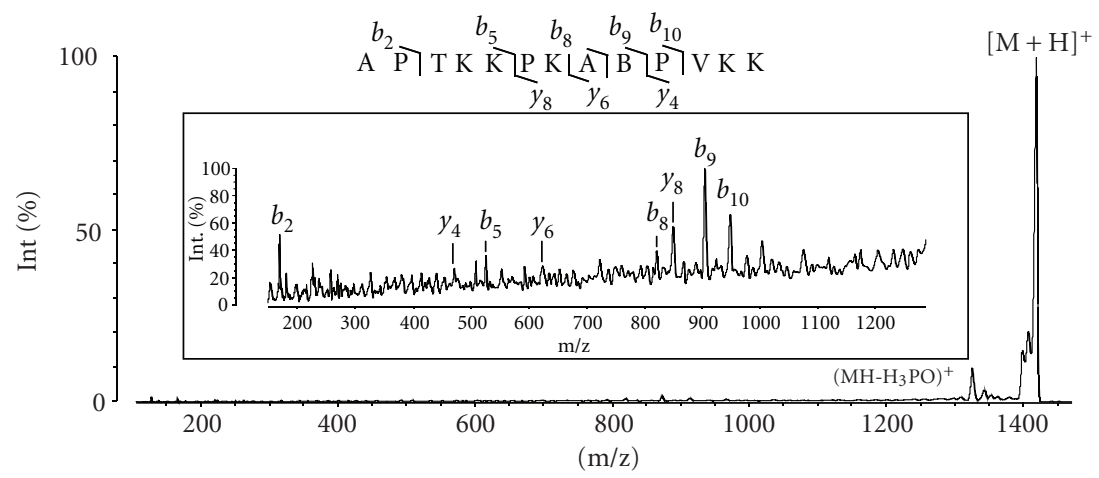

(c)

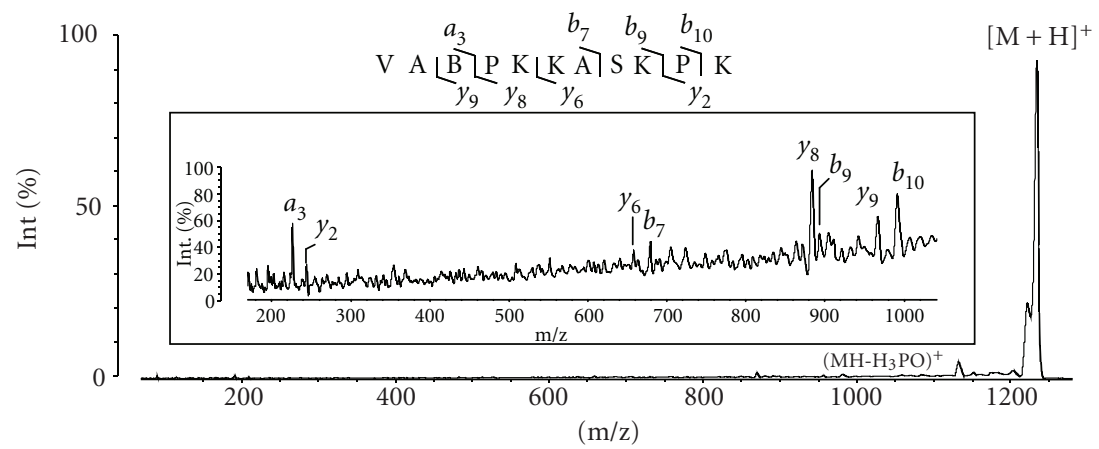

(d)

Figure 7: The MALDI MS analysis of phosphopeptides from CDK1-treated human histone H1. MS spectra of the tryptic peptides from CDK1-treated histone H1 with the untreated (Figure 7(a)) and alkaline phosphatase-treated histone H1 (Figure 7(b)). The two panels on the right show the magnified spectra to indicate the two phosphopeptides labeled with asterisks $(*)$. The MALDI-TOF/TOF-MS analysis of phosphorylation sites on the two monophosphopeptides VApTPKKASKPK, m/z 1234.7 (c), APTKKPKApTPVKK, m/z 1473.9 (d) from the CDK1-treated human histone $\mathrm{H} 1$. The neutral-loss peak of phosphopeptide was noted as $\left[\mathrm{MH}-\mathrm{H}_{3} \mathrm{PO}_{4}\right]^{+}$. The fragment patterns of peptides were shown in the magnified MS/MS spectra. " $\mathrm{B}$ " in the amino sequence indicates a dehydroamino-2-butyric acid residue converted from a phosphothreonine residue by beta-elimination of $\mathrm{H}_{3} \mathrm{PO}_{4}$. All the spectra were detected using DHAP/DAHC matrix in the positive mode. 
change of the DHAP/DAHC ratio. We calculated the Bull and Breese Index (BB Index) [19] of these peptides, an index generally used to reflect the hydrophobic/hydrophilic nature of peptides. Higher BB Index values are associated with more hydrophilic peptides. For the phosphopeptide, we calculated the BB Index by replacing the phosphoserine or phosphothreonine residues with glutamic acid [19]. By comparing the BB Index of peptides listed in Table 1, we found that the phosphopeptides were nearly hydrophilic peptides with high $\mathrm{BB}$ Indices and that the nonphosphopeptides for which signal intensity was weakened at low DHAP/DAHC ratio were highly hydrophobic with lower BB Index (T4: -5530, T11:-6480, T18:-6900). The nonphosphopeptide T10 for which the ion signal was enhanced at lower ratios of DHAP/DAHC was found to be slightly hydrophobic with a BB Index of -750 . These findings indicated that the optimal DAHC/DHAP matrix tended to detect more hydrophilic peptides. Most phosphopeptides are hydrophilic, so we propose that the low ratio of DHAP/DAHC matrix could increase cocrystallization between matrix and phosphopeptides and further improve ionization efficiency of phosphopeptide in MALDI MS.

3.2. Sample Homogeneity. In this study, the phosphopeptide pT (250 fmol on target) was used to investigate the sample homogeneity of optimized DHAP/DAHC matrix, and for a comparison, we chose matrix combination of $2,5-\mathrm{DHB}$ with $1 \%$ phosphoric acid, a currently popular matrix used for detection of phosphopeptide [30-32]. It was apparent from the photographs of the MALDI sample spots that there was a dramatic difference between the optimized DHAP/DAHC (Figure 2(a)) and DHB/PA (Figure 2(b)). Small crystals formed in a DHAP/DAHC sample spot while larger, needle-like crystals formed in a DHB/PA sample spot. Furthermore, $196(14 \times 14)$ positions on the target were measured covering the crystalline layer for a full overview (Figure 2(e)), and the MS signal intensities of pT were monitored. The majority of the outer zones of the crystalline samples exhibited high signal intensities, except for a small center zone when DHAP/DAHC used as matrix (Figure 2(c)). In comparison, the crystalline DHB/PA matrix exhibited the hot-spot phenomenon, signals were primarily observed in the narrow, inhomogeneous ring at the edge of the sample layer, and the inner position almost did not exhibit any signal (Figure $2(\mathrm{~d})$ ). Therefore, the samples prepared with DHAP/DAHC were more homogeneous than those prepared with a DHB/PA matrix, reducing the need for time-consuming hot-spot searches. Based on the sample homogeneity of DHAP/DAHC and DHB/PA, in the next experiments, the data acquisition with DHAP/DAHC was carried out by randomly measuring the outer zone of the sample layer, while data were acquired from the DHB/PA matrix by searching for hot spots of sample.

3.3. Sensitivity Measurement of Phosphopeptides with the DHAP/DAHC Matrix. In order to further evaluate the measurement sensitivity of phosphopeptides with the optimal
DHAP/DAHC matrix, the tryptic digest of $\alpha$-casein and $\beta$ casein with $2.5-250 \mathrm{fmol}$ on the target were detected by MALDI-TOF MS in both positive and negative ion mode. Figure 3 shows that all eleven phosphopeptides from $\alpha$ casein and $\beta$-casein could be detected with signal-to-noise $(\mathrm{S} / \mathrm{N})$ ratios greater than 3 at the low amount of $12.5 \mathrm{fmol}$ in the positive mode (a) and (b) and detected at a lower amount of $5 \mathrm{fmol}$ in the negative mode (c) and (d). The monophosphopeptides ( $\mathrm{T} \alpha 1$ and $\mathrm{T} \beta 1$ ) could still be observed at $2.5 \mathrm{fmol}$ on target in negative ion mode (Figure 3(c)). Phosphopeptides generally exhibited better $\mathrm{S} / \mathrm{N}$ ratios in the MS spectra in negative ion mode than that in positive ion mode, although, consistent with previous reports [19], the absolute signal intensity of phosphopeptide was usually lower in negative ion mode. Because all tryptic phosphopeptides from $\alpha$-casein and $\beta$-casein are serine-phosphorylated peptides, a threonine-phosphorylated peptide $\mathrm{pT}$ and a tyrosinephosphorylated peptide pY (2.5-250 fmol on target) were used to investigate whether the DHAP/DAHC matrix had a generic nature in detection of phosphopeptides. As expected, pT and pY could be readily detected down to $5 \mathrm{fmol}$ on the target in both positive and negative modes (Figure 4).

We further directly compared optimized DHAP/DAHC to $\mathrm{DHB} / \mathrm{PA}$. Tryptic digests of $\alpha$-casein and $\beta$-casein (12.5$250 \mathrm{fmol}$ on target) were detected using these two matrices (Figure 5). When using the DHAP/DAHC matrix, all the phosphopeptides could readily be detected at three different sample amounts. By comparison, when DHB/PA was used as the matrix, although all the phosphopeptides in $250 \mathrm{fmol}$ digest (on target) could be detected with $\mathrm{DHB} / \mathrm{PA}$, the amount of digest went down to $50 \mathrm{fmol}$ (on the target), and only $\mathrm{T} \alpha 1, \mathrm{~T} \alpha 2, \mathrm{~T} \alpha 3, \mathrm{~T} \alpha 6, \mathrm{~T} \beta 1$, and $\mathrm{T} \beta 2$ could be observed. Furthermore, when the amount of digest went down to $12.5 \mathrm{fmol}$, only $\mathrm{T} \alpha 1$ and $\mathrm{T} \beta 1$ were detected, with weak signals. Comparing these results, it was clear that compared with DHB/PA, the optimized DHAP/DAHC had comparative sensitivity in the detection of singly phosphorylated peptides ( $\mathrm{T} \alpha 1$ and $\mathrm{T} \beta 1$ ), but greater sensitivity in the detection of multiply phosphorylated peptides, such as $\mathrm{T} \beta 2$ (4p) and $\mathrm{T} \alpha 4$ (5p). Herein, we suggest that sample should be analyzed immediately after sample preparation when optimized DHAP/DAHC matrix used, as the crystals might not last too long under atmospheric conditions and in vacuo.

3.4. Sequencing of Phosphopeptides with High Energy CID $M S / M S$. We employed the AXIMA-TOF ${ }^{2}$ MALDI mass spectrometer (Shimadzu/Kratos, Manchester, UK) to perform high energy CID MS/MS of phosphopeptides to characterize phosphorylation sites. Figure 6 shows the MS/MS spectra of three phosphopeptides T $\beta 1, \mathrm{~T} \alpha 3$, and T $\beta 2$ from $\alpha$ casein and $\beta$-casein ( $250 \mathrm{fmol}$ on target) with DHAP/DAHC as matrix. The number of $[\mathrm{MH}-98]^{+}$or $[\mathrm{MH}-80]^{+}$peaks in the MS/MS spectra indicated the number of phosphate groups in each phosphorylated peptide, and the fragment ions including $a, b$, and $y$ ions clearly show the phosphorylation sites of the phosphopeptides $\mathrm{T} \beta 1, \mathrm{~T} \alpha 3$, and $\mathrm{T} \beta 2$. These results demonstrated the feasibility of MALDI-TOF/TOF 
MS with the DHAP/DAHC matrix for the analysis of the phosphorylation sites of phosphopeptides, including singly and multiply phosphorylated peptides.

\subsection{Application of the Optimized DHAP/DAHC Matrix in} the Characterization of Phosphorylated Human Histone H1. Further investigating the practicality of the DHAP/DAHC matrix, we used this approach to characterize human histone H1 phosphorylated with CDK1. Experimentally, phosphorylated histone $\mathrm{H} 1$ was separated by SDS-PAGE and ingel digested by trypsin. In order to generate appropriate peptide sizes for MALDI-MS analysis, since the sequence of histone proteins is known to contain many lysine and arginine residues, the trypsin digestion time was optimized and reduced to 1 hour. Figure 7(a) shows the results of the MALDI-TOF-MS analysis of the tryptic digest of the phosphorylated histone $\mathrm{H} 1$ without the desalting step, directly detected with the optimized DHAP/DAHC matrix in positive ion mode. After performing data processing using the on-line MASCOT search engine, 31 peptides, including 2 possible phosphopeptides (in Figure 7(a) labeled with “*”), were assigned to human histone $\mathrm{H} 1$, and $81 \%$ of the sequence was covered. These two peptides corresponded to the two monophosphopeptides of histone $\mathrm{H} 1$, comprising amino acids 117-127 (VATPKKA타K, $1234.7 \mathrm{~m} / \mathrm{z}$; possible phosphorylation sites indicated with underlined letters) and 133-145 (APTKKPKATPVKK, $1473.9 \mathrm{~m} / \mathrm{z}$ ). The tryptic sample of $\mathrm{H} 1$ was also analyzed with $\mathrm{DHB} / \mathrm{PA}$, but the two peaks of the phosphopeptide were lower than those in Figure 7(a) (see Figure S1). To validate the MASCOT searching result for the two phosphorylated peptides, we compared the mass spectra of tryptic peptides of $\mathrm{H} 1$ before and after treatment with alkaline phosphatase, which can cleave the phosphate group from phosphopeptides. It was clear that the two peptide peaks (marked with asterisks in Figure $7(\mathrm{a})$ ) had disappeared and two new peaks with a mass shift of $80 \mathrm{Da}\left(\mathrm{HPO}_{3}=80 \mathrm{Da}\right)$ were visible (Figure $7(\mathrm{~b})$ ). This demonstrated that the two peptides (marked with asterisks in Figure 7(a) were singly phosphorylated peptides. To identify the phosphorylation sites, we used MALDITOF/TOF MS to perform MS/MS analysis of the two peptides (Figure 7(c) and 7(d)). Only one neutral-loss peak corresponding to $\left[\mathrm{MH}-\mathrm{H}_{3} \mathrm{PO}_{4}\right]^{+}$indicated that both phosphopeptides were monophosphopeptides. After a MASCOT search, the phosphorylation sites of the two phosphopeptides were determined to be APTKKPKApTPVKK (pT indicating the phosphothreonine) and VApTPKKASKPK with ion scores of 53 and 50, respectively (scores exceeding 38 were accepted as significant matches).

In general, the protein phosphorylation sites by a particular protein kinase shared a set of "consensus sequence", which is necessary and sufficient for recognition by the kinase [33]. To further validate the phosphorylation sites of histone H1 identified with MALDI-TOF/TOF MS, we compared the two phosphorylated peptide sequences with the consensus sequence $\mathrm{pS} / \mathrm{pT}-\mathrm{P}-\mathrm{X}-\mathrm{R} / \mathrm{K}$, most frequently recognized by CDK1 [34, 35], and found that both phosphopeptide sequences were consistent with the consensus sequence.
These results thus demonstrate that the DHAP/DAHC matrix was robust and effective in analyzing the protein phosphorylation of the biological sample by MALDI-MS.

\section{Conclusion}

By optimizing the matrix solution composition, we found that a low ratio of DHAP/DAHC formulation was more efficient in detecting phosphopeptides than earlier protocols. Compared with DHB/PA, the optimized DHAP/DAHC exhibited higher sample homogeneity, and the phosphopeptides in tryptic digests of $\alpha$ - and $\beta$-casein without desalting and phosphopeptide enrichment could be measured with a higher sensitivity. Further, the optimized DHAP/DAHC showed high energy CID MS/MS analysis of the phosphorylation sites of phosphopeptides, including multiple phosphorylated peptides, and we successfully applied this method to the characterization of the phosphorylation sites of CDK1-treated human histone H1.

\section{Acknowledgments}

This research was supported by the National Basic Research Program of China (973) (Grant nos. 2004CB720004, and 2010CB833703), and the National Natural Science Foundation of China (Grant nos. 90919047, and 30670587). The authors are very grateful to Professor Chuanmao Zhang and Dr. Qiang Chen from the State Key Laboratory of Biomembrane and Membrane Bio-engineering, College of Life Sciences, Peking University for help preparing the sample of human histone $\mathrm{H} 1$ phosphorylated by CDK1.

\section{References}

[1] J. Posada and J. A. Cooper, "Molecular signal integration. Interplay between serine, threonine, and tyrosine phosphorylation," Molecular Biology of the Cell, vol. 3, no. 6, pp. 583-592, 1992.

[2] T. Hunter, "Protein kinases and phosphatases: the yin and yang of protein phosphorylation and signaling," Cell, vol. 80 , no. 2 , pp. 225-236, 1995.

[3] T. Hunter, "Signaling —2000 and beyond," Cell, vol. 100, no. 1, pp. 113-127, 2000.

[4] P. Cohen, "The regulation of protein function by multisite phosphorylation-a 25 year update," Trends in Biochemical Sciences, vol. 25, no. 12, pp. 596-601, 2000.

[5] M. Mukherji, "Phosphoproteomics in analyzing signaling pathways," Expert Review of Proteomics, vol. 2, no. 1, pp. 117128, 2005.

[6] T.-T. Yip and T. W. Hutchens, "Mapping and sequence-specific identification of phosphopeptides in unfractionated protein digest mixtures by matrix-assisted laser desorption/ionization time-of-flight mass spectrometry," FEBS Letters, vol. 308, no. 2, pp. 149-153, 1992.

[7] A. G. Craig, C. A. Hoeger, C. L. Miller, T. Goedken, J. E. Rivier, and W. H. Fischer, "Monitoring protein kinase and phosphatase reactions with matrix-assisted laser desorption/ionization mass spectrometry and capillary zone electrophoresis: comparison of the detection efficiency of peptidephosphopeptide mixtures," Biological Mass Spectrometry, vol. 23, no. 8, pp. 519-528, 1994. 
[8] P.-C. Liao, J. Leykam, P. C. Andrews, D. A. Gage, and J. Allison, "An approach to locate phosphorylation sites in a phosphoprotein: mass mapping by combining specific enzymatic degradation with matrix-assisted laser desorption/ionization mass spectrometry," Analytical Biochemistry, vol. 219, no. 1, pp. 9-20, 1994.

[9] R. S. Annan and S. A. Carr, "Phosphopeptide analysis by matrix-assisted laser desorption time-of-flight mass spectrometry," Analytical Chemistry, vol. 68, no. 19, pp. 34133421, 1996.

[10] S. A. Carr, M. J. Huddleston, and R. S. Annan, "Selective detection and sequencing of phosphopeptides at the femtomole level by mass spectrometry," Analytical Biochemistry, vol. 239, no. 2, pp. 180-192, 1996.

[11] M. Busman, K. L. Schey, J. E. Oatis Jr., and D. R. Knapp, "Identification of phosphorylation sites in phosphopeptides by positive and negative mode electrospray ionization-tandem mass spectrometry," Journal of the American Society for Mass Spectrometry, vol. 7, no. 3, pp. 243-249, 1996.

[12] R. S. Annan and S. A. Carr, "The essential role of mass spectrometry in characterizing protein structure: mapping posttranslational modifications," Protein Journal, vol. 16, no. 5, pp. 391-402, 1997.

[13] K. Stühler and H. E. Meyer, "MALDI: more than peptide mass fingerprints," Current Opinion in Molecular Therapeutics, vol. 6, no. 3, pp. 239-248, 2004.

[14] M. Khan, H. Takasaki, and S. Komatsu, "Comprehensive phosphoproteome analysis in rice and identification of phosphoproteins responsive to different hormones/stresses," Journal of Proteome Research, vol. 4, no. 5, pp. 1592-1599, 2005.

[15] G. Yan, L. Li, Y. Tao, et al., "Identification of novel phosphoproteins in signaling pathways triggered by latent membrane protein 1 using functional proteomics technology," Proteomics, vol. 6, no. 6, pp. 1810-1821, 2006.

[16] H. Steen, J. A. Jebanathirajah, J. Rush, N. Morrice, and M. W. Kirschner, "Phosphorylation analysis by mass spectrometry: myths, facts, and the consequences for qualitative and quantitative measurements," Molecular and Cellular Proteomics, vol. 5, no. 1, pp. 172-181, 2006.

[17] P. Cohen, "The role of protein phosphorylation in human health and disease," European Journal of Biochemistry, vol. 268, no. 19, pp. 5001-5010, 2001.

[18] D. Arnott, M. A. Gawinowicz, R. A. Grant, et al., "ABRFPRG03: phosphorylation site determination," Journal of Biomolecular Techniques, vol. 14, no. 3, pp. 205-215, 2003.

[19] S. Kjellström and O. N. Jensen, "Phosphoric acid as a matrix additive for MALDI MS analysis of phosphopeptides and phosphoproteins," Analytical Chemistry, vol. 76, no. 17, pp. 5109-5117, 2004.

[20] A. Tholey, "Ionic liquid matrices with phosphoric acid as matrix additive for the facilitated analysis of phosphopeptides by matrix-assisted laser desorption/ionization mass spectrometry," Rapid Communications in Mass Spectrometry, vol. 20, no. 11, pp. 1761-1768, 2006.

[21] H. Zhou, S. Xu, M. Ye, et al., "Zirconium phosphonatemodified porous silicon for highly specific capture of phosphopeptides and MALDI-TOF MS analysis," Journal of Proteome Research, vol. 5, no. 9, pp. 2431-2437, 2006.

[22] X. Yang, H. Wu, T. Kobayashi, R. J. Solaro, and R. B. Van Breemen, "Enhanced ionization of phosphorylated peptides during MALDI TOF mass spectrometry," Analytical Chemistry, vol. 76, no. 5, pp. 1532-1536, 2004.
[23] J. M. Asara and J. Allison, "Enhanced detection of phosphopeptides in matrix-assisted laser desorption/ionization mass spectrometry using ammonium salts," Journal of the American Society for Mass Spectrometry, vol. 10, no. 1, pp. 35-44, 1999.

[24] J. J. Gorman, B. L. Ferguson, and T. B. Nguyen, "Use of 2,6-dihydroxyacetophenone for analysis of fragile peptides, disulphide bonding and small proteins by matrix-assisted laser desorption/ionization," Rapid Communications in Mass Spectrometry, vol. 10, no. 5, pp. 529-536, 1996.

[25] T. Nabetani, K. Miyazaki, Y. Tabuse, and A. Tsugita, "Analysis of acidic peptides with a matrix-assisted laser desorption/ionization mass spectrometry using positive and negative ion modes with additive monoammonium phosphate," Proteomics, vol. 6, no. 16, pp. 4456-4465, 2006.

[26] C.-F. Xu, Y. Lu, J. Ma, M. Mohammadi, and T. A. Neubert, "Identification of phosphopeptides by MALDI Q-TOF MS in positive and negative ion modes after methyl esterification," Molecular and Cellular Proteomics, vol. 4, no. 6, pp. 809-818, 2005.

[27] C.-F. Xu, H. Wang, D. Li, X.-P. Kong, and T. A. Neubert, "Selective enrichment and fractionation of phosphopeptides from peptide mixtures by isoelectric focusing after methyl esterification," Analytical Chemistry, vol. 79, no. 5, pp. 20072014, 2007.

[28] V. J. Nesatyy, A. Dacanay, J. F. Kelly, and N. W. Ross, "Microwave-assisted protein staining: mass spectrometry compatible methods for rapid protein visualisation," Rapid Communications in Mass Spectrometry, vol. 16, no. 4, pp. 272280, 2002.

[29] S. L. Cohen and B. T. Chait, "Influence of matrix solution conditions on the MALDI-MS analysis of peptides and proteins," Analytical Chemistry, vol. 68, no. 1, pp. 31-37, 1996.

[30] T. E. Thingholm, T. J. D. Jørgensen, O. L. Jensen, and M. R. Larsen, "Highly selective enrichment of phosphorylated peptides using titanium dioxide," Nature Protocols, vol. 1, no. 4, pp. 1929-1935, 2006.

[31] G. Luo, A. Gruhler, Y. Liu, O. N. Jensen, and R. C. Dickson, "The sphingolipid long-chain base-Pkh1/2-Ypk1/2 signaling pathway regulates eisosome assembly and turnover," Journal of Biological Chemistry, vol. 283, no. 16, pp. 10433-10444, 2008.

[32] C. Krintel, P. Osmark, M. R. Larsen, S. Resjö, D. T. Logan, and C. Holm, "Ser649 and Ser650 are the major determinants of protein kinase A-mediated activation of human hormonesensitive lipase against lipid substrates," PLoS ONE, vol. 3, no. 11, article e3756, 2008.

[33] P. J. Kennelly and E. G. Krebs, "Consensus sequences as substrate specificity determinants for protein kinases and protein phosphatases," Journal of Biological Chemistry, vol. 266, no. 24, pp. 15555-15558, 1991.

[34] L. M. Stevenson-Lindert, P. Fowler, and J. Lew, "Substrate specificity of CDK2-cyclin A: what is optimal?" Journal of Biological Chemistry, vol. 278, no. 51, pp. 50956-50960, 2003.

[35] Z. Songyang, S. Blechner, N. Hoagland, M. F. Hoekstra, H. Piwnica-Worms, and L. C. Cantley, "Use of an oriented peptide library to determine the optimal substrates of protein kinases," Current Biology, vol. 4, no. 11, pp. 973-982, 1994. 

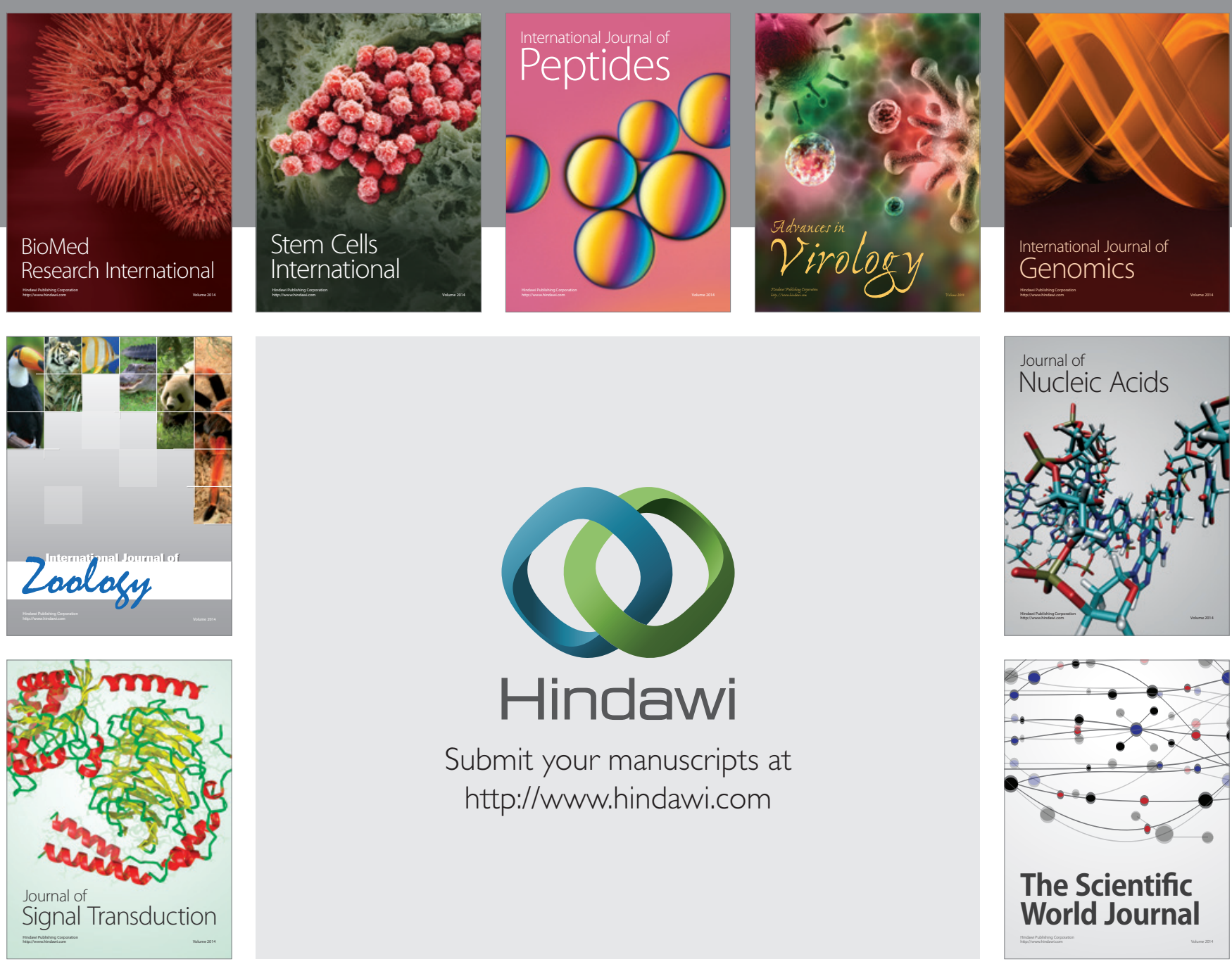

Submit your manuscripts at

http://www.hindawi.com
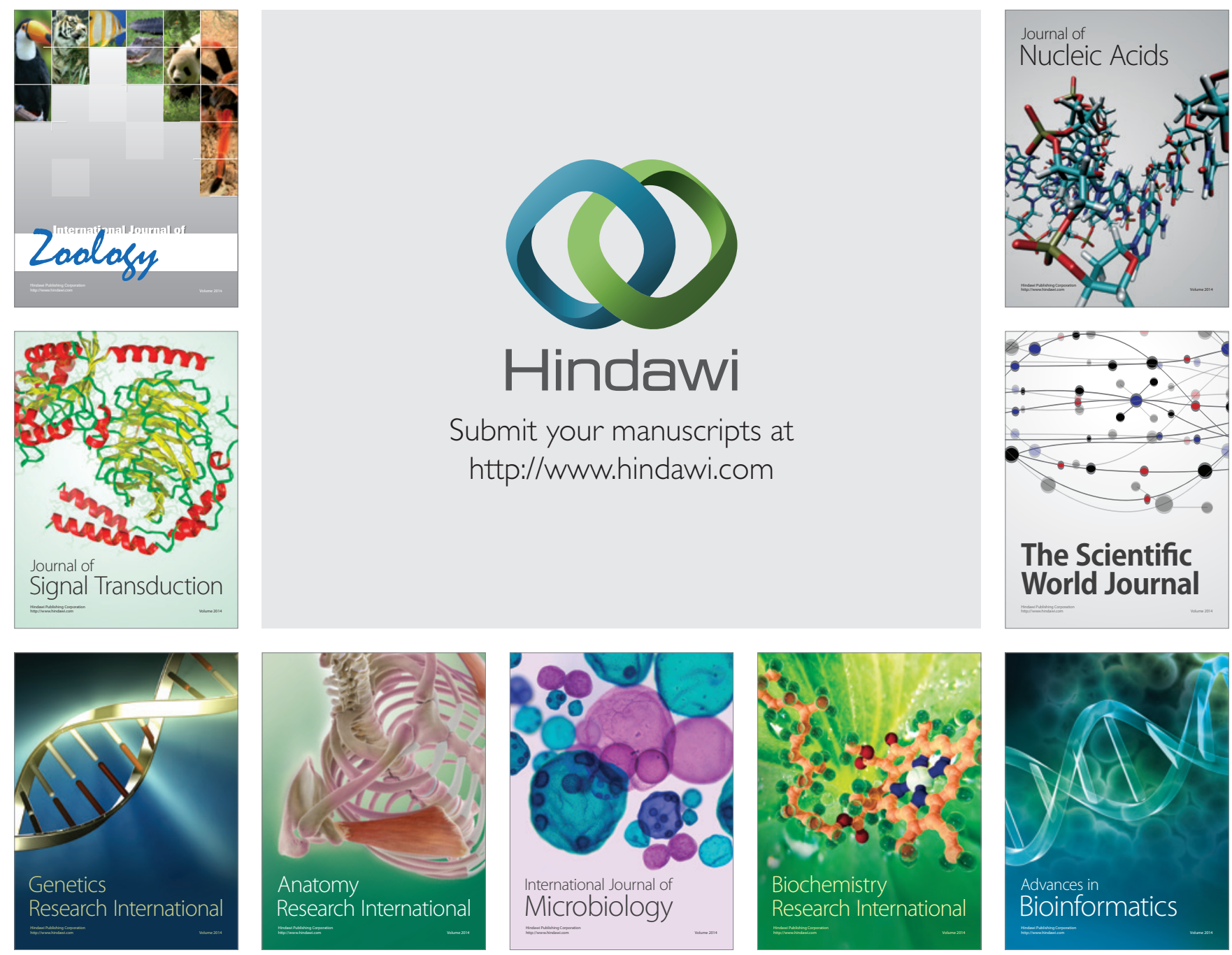

The Scientific World Journal
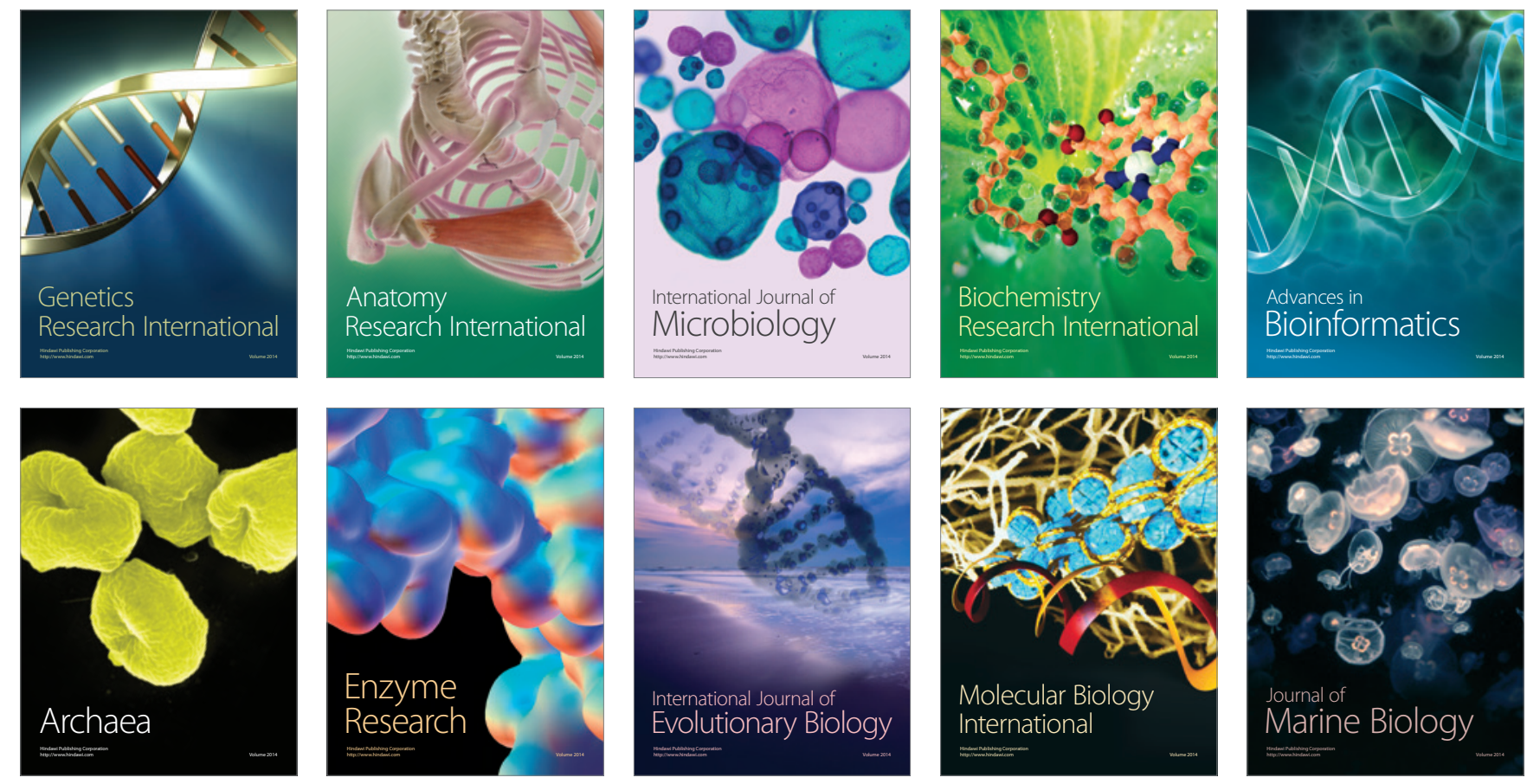